\title{
When and how to replace the aortic root in type A aortic dissection
}

\author{
Bradley G. Leshnower, Edward P. Chen \\ Division of Cardiothoracic Surgery, Joseph B. Whitehead Department of Surgery, Emory University School of Medicine, Atlanta, GA, USA \\ Correspondence to: Edward P. Chen, MD. Associate Professor, Director of Thoracic Aortic Surgery, Division of Cardiothoracic Surgery, Emory \\ University School of Medicine, 5665 Peachtree Dunwoody Road, Suite 200, Atlanta, GA 30342, USA. Email: epchen@emory.edu.
}

\begin{abstract}
Management of aortic root pathology during repair of acute type A aortic dissection (TAAD) requires a comprehensive evaluation of the patient's anatomy, demographics, comorbidities and physiologic status at the time of emergent operative intervention. Surgical options include conservative repair of the root (CRR) (with or without replacement of the aortic valve), replacement of the native valve and aortic root using a composite valve-conduit and valve sparing root replacement (VSRR). The primary objective of this review is to provide data for surgeons to aid in their decision-making process regarding management of the aortic root during repair of TAAD. No time or language restrictions were imposed and references of the selected studies were checked for additional relevant citations. Multiple retrospective reviews have demonstrated equivalent operative mortality between aortic root repair and replacement during TAAD. There is a higher incidence of aortic root reintervention with aortic root repair compared to aortic root replacement (ARR). Experienced, high-volume aortic centers have demonstrated the safety of VSRR in young, hemodynamically stable patients presenting with TAAD. In conclusion, aortic root repair can safely be performed in the vast majority of patients with TAAD. Despite the increased surgical complexity, ARR does not increase operative mortality and improves the freedom from root reintervention. VSRR can be performed in highly selected populations of patients with TAAD with durable mid-term valve function.
\end{abstract}

Keywords: Aortic dissection; aortic root replacement (ARR)

Submitted Jan 10, 2016. Accepted for publication Feb 28, 2016.

doi: 10.21037/acs.2016.03.15

View this article at: http://dx.doi.org/10.21037/acs.2016.03.15

\section{Introduction}

Contemporary surgical therapy for acute type A aortic dissection (TAAD) involves excision of the primary intimal tear, replacement of the ascending aorta, correction of aortic valve dysfunction, treatment of aortic root pathology, and proximal or total arch reconstruction. Options for the management of the aortic root in TAAD include root and valve replacement with a composite valvegraft conduit (CVG), valve sparing root replacement (VSRR) or conservative repair of the aortic root (CRR), with or without aortic valve replacement. In order to provide the optimal treatment for each individual patient, a comprehensive evaluation of the patient's anatomy, demographics, comorbidities and physiologic status at the time of surgery must be performed.

The initial decision regarding the management of the aortic root in TAAD is whether to repair or replace the dissected sinus segments. CRR involves the preservation and fortification of the dissected sinus segments with either resuspension of the native aortic valve commissural posts or prosthetic aortic valve replacement. Aortic root replacement (ARR) requires excision of the dissected sinus segments and either replacement or preservation of the aortic valve with reimplantation of the coronary arteries. The standard indications for ARR in the setting TAAD are extensive tissue destruction, the presence of a concomitant aortic root aneurysm $\geq 4.5 \mathrm{~cm}$, or a known connective tissue disorder (1).

The purpose of this manuscript is to review the current techniques and data in the literature regarding the various options for aortic root intervention in the setting of TAAD. This review will serve as an aid to surgeons in the management of the acutely dissected aortic root. 

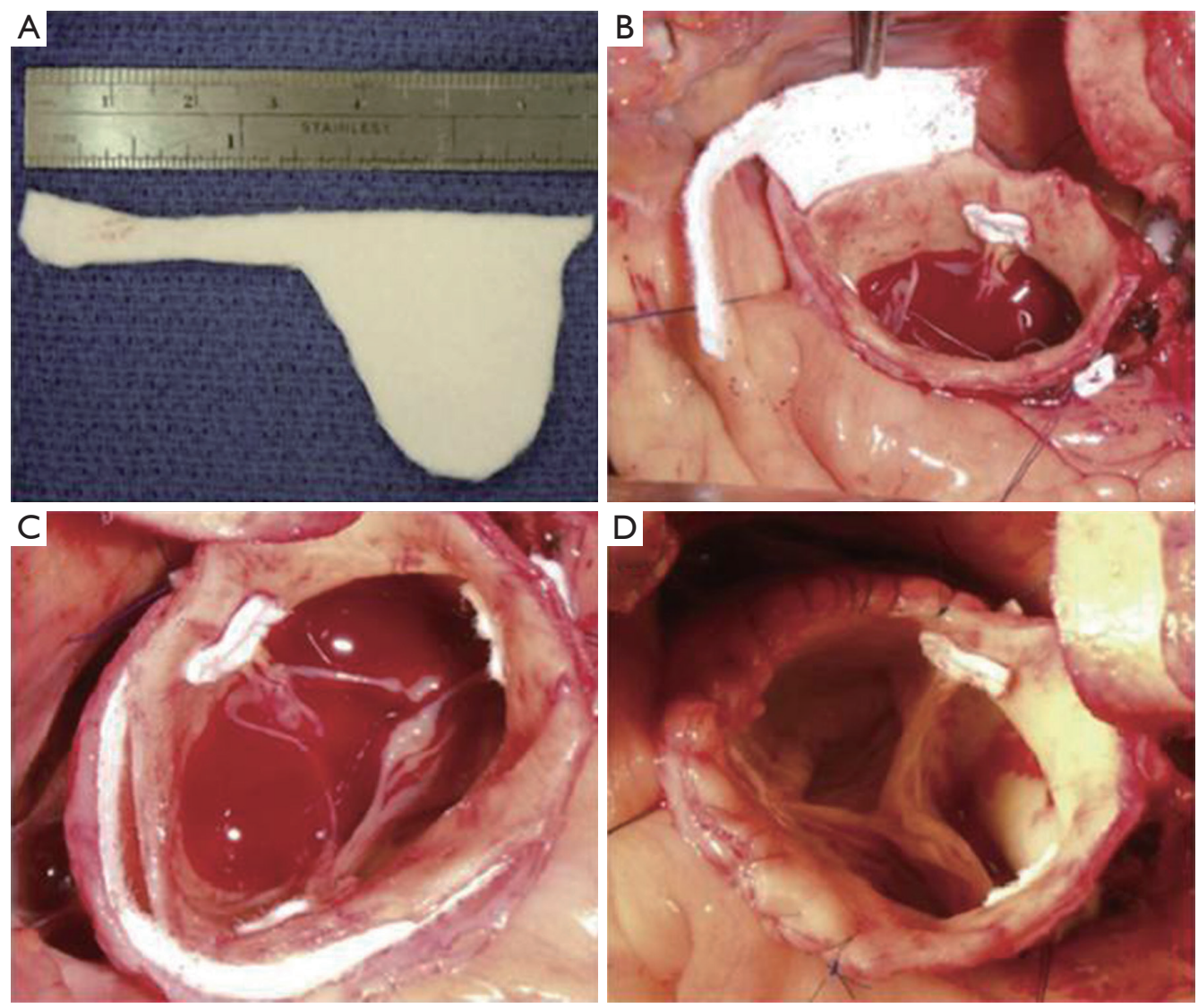

Figure 1 The "neomedia" technique. Insertion of a "neo-sinus" segment (4).

\section{Results}

\section{Aortic root repair}

In the vast majority of patients presenting with TAAD, the aortic root can be repaired. The most common pathology observed is a primary intimal tear located in the ascending aorta with extension of the dissection flap into the noncoronary cusp, and relative preservation of the left and right coronary sinuses. Rarely are the aortic valve cusps or annulus impacted by the dissection process. Therefore, the aortic regurgitation associated with TAAD does not represent a primary leaflet problem; instead, it is due to disruption of the aortic wall architecture with concomitant "unhinging" of one or more of the aortic valve commissural posts. This pathologic process results in cusp prolapse and acute aortic regurgitation. Repair of the dissected sinus segments with resuspension of the aortic valve commissures typically restores aortic valve competency.

CRR offers several advantages over ARR. It is a simpler procedure that preserves the native sinus tissue, avoids manipulation of the coronary arteries and requires shorter myocardial ischemic and cardiopulmonary bypass times. Avoidance of a valve replacement, in the setting of normal cusps, significantly reduces the likelihood of reintervention on the aortic valve or the need for lifelong anticoagulation, as is the case with implantation of a bioprosthetic or mechanical valve. The elimination of anticoagulation in patients with TAAD may promote false lumen thrombosis, reduce the need for reintervention on the distal aorta, and improve long-term survival $(2,3)$.

Multiple root repair techniques using prosthetic and biologic materials have been reported (4-9). The two most commonly described methods involve fortification of the aortic wall using Teflon felt and/or biologic glue. The Teflon felt "neomedia" technique refers to the insertion of this material between the dissected intimal and adventitial layers (Figure 1). The "sandwich" technique consists of internal and external layers of felt that are incorporated into the supracoronary aortic graft anastomosis (Figure 2). Biologic glues have been used as either an adjunct to the 


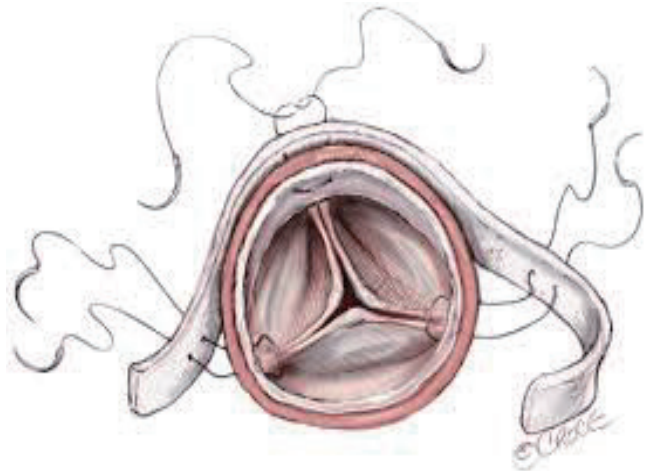

Figure 2 The "sandwich" technique: internal and external fortification of the aortic wall with Teflon felt (10).

felt techniques or as stand-alone agent to reapproximate the dissected layers of the aortic wall.

Based upon data from several large volume series, CRR is a safe procedure with acceptable operative mortality rates. The efficacy and durability of the different CRR techniques can be judged by the incidence of reintervention required to address severe aortic regurgitation and/or aneurysmal degeneration of the root. The largest experience with CRR was reported by the University of Pennsylvania group. These authors described a 20-year experience with a standardized repair approach consisting of Teflon felt tailored to the shape of a "neo-sinus" segment that is inserted between the intima and adventitia of all dissected sinuses to recreate a medial layer. The Teflon felt was secured in place with a running 5-0 polyprolene suture and small amounts of Bioglue (CryoLife Inc., Kennesaw, GA, USA) (Figure 1). In their series of 489 patients who underwent CRR during TAAD, operative mortality was $11 \%$ and the freedom from root reoperation was $96 \%$, $92 \%$ and $89 \%$ at 1,10 and 15 years respectively (4). Results with the "sandwich" technique of CRR were described by Mazzucotelli and colleagues. In their series of 80 patients, these authors reported an operative mortality of $29 \%$, and a freedom from reoperation on the aortic root of $83 \%$ at 5 years and $79 \%$ at 15 years (6).

In contrast to the CRR techniques using felt, the results of using biologic glue as a stand-alone technique demonstrate acceptable operative results, but less durable long-term outcomes. Casselman and colleagues described their CRR technique using gelatin-resorcinolformaldehyde-glue (GRF-glue, Fii, Saint-Just Malmont, France) to reapproximate the dissected layers of the aortic wall in a series of 246 patients with TAAD. CRR was performed in 121 (49\%) patients in this series. The operative mortality for the entire series $(\mathrm{n}=246)$ was $21.5 \%$ and the freedom from reoperation on the aortic root was $95 \%$ at one year and $69 \%$ at ten years (7). Several other series of CRR report unacceptably high rates of root reoperation with GRF glue alone. In these reports, GRF glue has been associated with tissue necrosis, aortic root redissection, and pseudoaneurysm formation (11-14). Histologic investigations at the time of reintervention have specifically identified the toxic effects of GRF upon aortic smooth muscle cells and elastin $(15,16)$. Bioglue, a newer agent that is composed of purified bovine serum albumin and glutaraldehyde, has replaced GRF. Bioglue appears to be less toxic, but long-term data is lacking. Rylski and colleagues reported on their mid-term experience with 97 patients who underwent CRR with the application of Bioglue alone. Operative mortality in this series was $8 \%$ and the freedom from reoperation on the aortic root at a median follow-up of 34 months was $90 \%$ (17).

\section{Aortic root replacement (ARR)}

ARR with a CVG in patients with TAAD is a significantly more complex procedure than CRR for multiple reasons. As stated previously, root replacement requires longer myocardial ischemic and cardiopulmonary bypass times in patients who often present with end-organ malperfusion or cardiogenic shock. Frequently the coronary artery ostia are involved in the dissection process and must be repaired prior to reimplantation. This may require saphenous vein graft extensions or coronary artery bypass in the case of irreparable damage.

Despite the increased risk associated with ARR, several large series comparing CRR to ARR have demonstrated equivalent operative mortality with a lower incidence of aortic root reoperation following ARR $(8,18,19)$. The Mount Sinai group reviewed their experience with CRR compared to ARR in 162 patients with TAAD. Operative mortality was $12.3 \%$ in each group, and the ten year survival was $65 \%$ in the ARR group and $55 \%$ in the CRR group $(\mathrm{P}=0.48)$. There was a trend towards a higher incidence of root reoperation in the repair group $(\mathrm{CRR} n=4 v s$. ARR $\mathrm{n}=0 ; \mathrm{P}=0.08)(18)$. The Hannover group reported similar results in their series of 295 patients with TAAD. Operative mortality (CRR 26\% vs. ARR 28\%; $\mathrm{P}=0.053$ ), and five year survival (CRR $80 \%$ vs. ARR $85 \%$; $\mathrm{P}=0.61$ ) were equivalent, but there was a significantly higher incidence of aortic root reoperation in the repair group (CRR 22\%vs. ARR 
$6.3 \% ; \mathrm{P}=0.005)$ (8). The largest comparison of CRR vs. ARR comes from the International Registry of Acute Aortic Dissection (IRAD). This report compared 699 patients who received ARR to 1,296 patients who received CRR. Operative mortality (CRR 18\% vs. ARR $21.3 \%$; $\mathrm{P}=0.07$ ) and 3-year survival (CRR 91.6\% vs. ARR 92.5\%; $\mathrm{P}=0.62$ ) were no different between the groups. The freedom from root reintervention at three years was $99 \%$ in both groups $(\mathrm{P}=0.77)$. This large study confirms the hypothesis that ARR does not increase operative mortality compared to CRR. Although these mid-term results did not show a higher reintervention rate with CRR, longer-term followup is necessary to evaluate the durability of root repair (8).

The alternative procedure to CRR and ARR that has been used more recently in patients with TAAD is VSRR with the reimplantation technique. Early experience with VSRR using the remodeling technique in TAAD demonstrated a high failure rate and, as a result, the remodeling technique has been abandoned in this clinical setting (20). Therefore this discussion will focus on the reimplantation technique.

In this procedure the aortic sinus segments are excised leaving a $2-3 \mathrm{~mm}$ remnant of aortic wall around the annulus. The remnant aorta and annulus are subsequently reimplanted into an appropriately sized Dacron graft that is based upon the height of the native aortic valve cusps. Originally designed as an alternative to ARR for the treatment of aortic root aneurysms, VSRR is an excellent option for hemodynamically stable, young patients with TAAD who present with concomitant aortic root aneurysms, extensive root destruction or connective tissue disorders with normal aortic valve cusps. In contrast to patients with chronic aortic regurgitation and altered aortic valve cusps, patients with TAAD typically have normal aortic valve cusps, as the tissue pathology is limited to the aortic wall. Any degree of valve incompetence can frequently be corrected with reimplantation of the aortic remnant into the properly sized graft without the additional need for cusp adjustment or manipulation.

VSRR is a more complex procedure than ARR. The surgical dissection required to perform this procedure is more extensive, requiring circumferential separation of the native aortic root from all surrounding structures to a level of approximately $2 \mathrm{~mm}$ below the nadir of the aortic. This can be challenging in the setting of TAAD when normal tissue planes are often disrupted by a significant amount of edema and hematoma. Furthermore, VSRR involves longer myocardial ischemic and cardiopulmonary bypass times that may require additional cross clamp time if significant aortic regurgitation is present upon separation from cardiopulmonary bypass.

Given the increased complexity and cardiopulmonary times associated with performing a VSRR in patients with TAAD, appropriate patient selection is essential to achieving a successful outcome. Patients presenting with significant cardiomyopathy, malperfusion syndromes or coronary artery disease are not suitable candidates for VSRR. Similarly, the increased risk of the procedure is not justified in patients $>65$ years of age, who could receive root replacement with a bioprosthetic CVG that would provide durable valve function well into their eighth decade of life. However, the presence of severe aortic regurgitation or bicuspid aortic valve anatomy does not represent absolute contraindications to VSRR in TAAD.

The existing data regarding performance of VSRR in TAAD is limited and has been reported by high volume aortic centers with significant experience with VSRR in the elective setting (19-23). These reports demonstrate durable valve function and no increase in operative mortality in relatively small cohorts of patients, thus reflecting the importance of careful patient selection. Our institutional experience with VSRR in TAAD at Emory supports these findings. From 2005-2013, 350 patients underwent surgical treatment of TAAD throughout Emory Healthcare. Within this cohort, ninety-eight (28\%) patients underwent ARR (ARR or VSRR), and the remainder received CRR. Forty-three (12\%) patients in this series underwent VSRR. The mean age of these patients was $46 \pm 10$ years and $>90 \%$ had a preoperative left ventricular ejection fraction of $\geq 55 \%$. Pre-operatively, twenty-seven (63\%) patients had $\geq$ moderate aortic regurgitation. All patients underwent hemiarch $(81 \%)$ or total arch replacement $(19 \%)$. The mean myocardial ischemia and cardiopulmonary bypass times were $237 \pm 45$ and $280 \pm 61$ minutes. Operative mortality was $4.7 \%$ and the mean follow up was $40 \pm 31$ months. At nine year follow-up, the freedom from $>$ mild aortic regurgitation was $94 \%$ and the freedom from aortic valve replacement was $100 \%$. There were no aortic root reinterventions and eight (19\%) distal aortic reinterventions in this series. These patients continue to be followed closely with annual surveillance echocardiograms and cross sectional imaging to monitor valve durability and the size of the residually dissected aorta (21).

\section{Conclusions}

In summary, the data presented above demonstrate that in the vast majority of patients, a strategy of root 
repair with preservation of the native sinus tissue can be accomplished with low operative mortality. The method of CRR significantly impacts long-term freedom from root reintervention, with Teflon felt-based techniques demonstrating superior durability compared to repair with biologic glue agents alone. A more aggressive strategy consisting of aortic root and valve replacement does not increase operative mortality and is associated with a lower late root reintervention rate. ARR is indicated in patients with concomitant aortic root aneurysms $\geq 4.5 \mathrm{~cm}$, extensive aortic root destruction or a history of a connective tissue disease. VSRR is also a feasible option in the management of the root in young, hemodynamically stable patients presenting with TAAD. Despite a significantly higher complexity, small series have demonstrated no difference in operative mortality compared to CRR or ARR. Surgeon experience and careful patient selection are vital to achieving successful outcomes with VSRR in TAAD.

\section{Acknowledgements}

None.

\section{Footnote}

Conflicts of Interest: The authors have no conflicts of interest to declare.

\section{References}

1. Hiratzka LF, Bakris GL, Beckman JA, et al. 2010 ACCF/AHA/AATS/ACR/ASA/SCA/SCAI/SIR/STS/ SVM guidelines for the diagnosis and management of patients with Thoracic Aortic Disease: a report of the American College of Cardiology Foundation/American Heart Association Task Force on Practice Guidelines, American Association for Thoracic Surgery, American College of Radiology, American Stroke Association, Society of Cardiovascular Anesthesiologists, Society for Cardiovascular Angiography and Interventions, Society of Interventional Radiology, Society of Thoracic Surgeons, and Society for Vascular Medicine. Circulation 2010;121:e266-369.

2. Park KH, Lim C, Choi JH, et al. Midterm change of descending aortic false lumen after repair of acute type I dissection. Ann Thorac Surg 2009;87:103-8.

3. Fattouch K, Sampognaro R, Navarra E, et al. Longterm results after repair of type a acute aortic dissection according to false lumen patency. Ann Thorac Surg 2009;88:1244-50.

4. Rylski B, Bavaria JE, Milewski RK, et al. Long-term results of neomedia sinus valsalva repair in 489 patients with type A aortic dissection. Ann Thorac Surg 2014;98:582-8; discussion 588-9.

5. Weinschelbaum EE, Schamun C, Caramutti V, et al. Surgical treatment of acute type A dissecting aneurysm, with preservation of the native aortic valve and use of biologic glue. Follow-up to 6 years. J Thorac Cardiovasc Surg 1992;103:369-74.

6. Mazzucotelli JP, Deleuze PH, Baufreton C, et al. Preservation of the aortic valve in acute aortic dissection: long-term echocardiographic assessment and clinical outcome. Ann Thorac Surg 1993;55:1513-7.

7. Casselman FP, Tan ES, Vermeulen FE, et al. Durability of aortic valve preservation and root reconstruction in acute type A aortic dissection. Ann Thorac Surg 2000;70:1227-33.

8. Di Eusanio M, Trimarchi S, Peterson MD, et al. Root replacement surgery versus more conservative management during type A acute aortic dissection repair. Ann Thorac Surg 2014;98:2078-84.

9. Zierer A, Voeller RK, Hill KE, et al. Aortic enlargement and late reoperation after repair of acute type A aortic dissection. Ann Thorac Surg 2007;84:479-86; discussion 486-7.

10. Okita Y, Okada K, Omura A, et al. Surgical techniques of total arch replacement using selective antegrade cerebral perfusion. Ann Cardiothorac Surg 2013;2:222-8.

11. von Oppell UO, Karani Z, Brooks A, et al. Dissected aortic sinuses repaired with gelatin-resorcin-formaldehyde (GRF) glue are not stable on follow up. J Heart Valve Dis 2002;11:249-57.

12. Suehiro K, Hata T, Yoshitaka H, et al. Late aortic root redissection following surgical treatment for acute type A aortic dissection using Gelatin-Resorcin-Formalin glue. Jpn J Thorac Cardiovasc Surg 2002;50:195-200.

13. Fukunaga S, Karck M, Harringer $W$, et al. The use of gelatin-resorcin-formalin glue in acute aortic dissection type A. Eur J Cardiothorac Surg 1999;15:564-9; discussion 570.

14. Hata H, Takano H, Matsumiya G, et al. Late complications of gelatin-resorcin-formalin glue in the repair of acute type A aortic dissection. Ann Thorac Surg 2007;83:1621-6.

15. Kunihara T, Iizuka K, Sasaki S, et al. Optimal proportions of gelatin-resorcin-formalin components in aortic surgery. Eur J Cardiothorac Surg 2009;36:962-6.

16. Suzuki S, Imoto K, Uchida K, et al. Aortic root necrosis after surgical treatment using gelatin-resorcinol- 
formaldehyde (GRF) glue in patients with acute type A aortic dissection. Ann Thorac Cardiovasc Surg 2006;12:333-40.

17. Rylski B, Beyersdorf F, Blanke P, et al. Supracoronary ascending aortic replacement in patients with acute aortic dissection type A: what happens to the aortic root in the long run? J Thorac Cardiovasc Surg 2013;146:285-90.

18. Halstead JC, Spielvogel D, Meier DM, et al. Composite aortic root replacement in acute type A dissection: time to rethink the indications? Eur J Cardiothorac Surg 2005;27:626-32; discussion 632-3.

19. Kallenbach K, Oelze T, Salcher R, et al. Evolving strategies for treatment of acute aortic dissection type A. Circulation 2004;110:II243-9.

20. Leyh RG, Fischer S, Kallenbach K, et al. High failure

Cite this article as: Leshnower BG, Chen EP. When and how to replace the aortic root in type A aortic dissection. Ann Cardiothorac Surg 2016;5(4):377-382. doi: 10.21037/ acs.2016.03.15 rate after valve-sparing aortic root replacement using the "remodeling technique" in acute type A aortic dissection. Circulation 2002;106:I229-33.

21. Leshnower BG, Myung RJ, McPherson L, et al. Midterm results of David V valve-sparing aortic root replacement in acute type A aortic dissection. Ann Thorac Surg 2015;99:795-800; discussion 800-1.

22. Erasmi AW, Stierle U, Bechtel JF, et al. Up to 7 years' experience with valve-sparing aortic root remodeling/ reimplantation for acute type A dissection. Ann Thorac Surg 2003;76:99-104.

23. Subramanian S, Leontyev S, Borger MA, et al. Valvesparing root reconstruction does not compromise survival in acute type A aortic dissection. Ann Thorac Surg 2012;94:1230-4. 